\title{
MULTIFIELD HYBRID METHOD APPLIED TO BUBBLE RISING AND COALESCENCE
}

\author{
R. DENEFLE ${ }^{1}$, S. MIMOUNI ${ }^{1}$, J-P. CALTAGIRONE ${ }^{2} \&$ S. VINCENT ${ }^{2}$ \\ ${ }^{1} \mathrm{EDF}$ R\&D, France. \\ ${ }^{2}$ 12M, Université Bordeaux 1, UMR5295, France.
}

\begin{abstract}
As a first step towards a new approach for the simulation of two-phase flows, the objective of this work is to check out the prediction of a model dedicated to large and distorted bubbles on twobubble coalescence cases. The multifield hybrid approach for two-phase flow modelling consists in dealing separately with the small and spherical bubbles, treated with a dispersed approach, and with the large and distorted ones, whose interface is located. The overall method relies also on an existing building block, consisting in a set of averaged models dedicated to dispersed bubbles, which has already been validated and has given a reasonable agreement with experimental data in cases where the spherical shape assumption is still valid for the dispersed phase. This paper aims to assess a conservative interface locating method based on level set adapted to two-fluid model for two-phase flows. The interface locating method is a part of a model dedicated to the simulation of large and distorted bubbles. At different liquid viscosities and densities, the model provides reasonable predictions of terminal velocities and shapes for rising bubble experiments.

The main outcome is the simulation of bubble coalescence where the distortion of the interface during the coalescence phenomenon is followed. The ability to simulate coalescence phenomena correctly is an important issue in the modelling of slug flows with interface locating methods.

Keywords: Bubble rising, coalescence, interface sharpening, multifield model, surface tension.
\end{abstract}

\section{INTRODUCTION}

Two-phase flows are featuring many industrial applications such as nuclear safety, heat exchangers and chemical reactors. Based on the interface structures, several flow regimes can be identified and commonly separated into three main groups; separated flows, dispersed flows and the last group would contain the flows such as bubbly annular, churn turbulent or slug flow. Although this classification has been experimentally confirmed since a few decades [1], the numerical simulation of complex two-phase flow regimes is still challenging and an universal model remains to be established.

The computation of two-phase flow simulation is a matter of choice, such as Eulerian or Lagrangian point of view, the number of fields, the use of space or time averages, the filtering of some space scales, the way the interfaces are dealt with, etc. It results into the following arborescence proposed by Bestion [2]:

- Phase or field averaging:

- Homogeneous for two-phase flow mixture.

- Two-fluid model.

- Multifield model.

- Filtering turbulent and two-phase intermittency scales:

- RANS (all turbulent scales are filtered).

- Two-phase LES (filtered under a characteristic size).

- DNS (all turbulent scales are simulated). 
- Treatment of interfaces:

- Interface tracking/capturing.

- Statistical treatment of interfaces.

- Identification of local interfaces.

- Characterization of the interface (colour function).

Depending on the studied phenomena, an approach may be more accurate than the other. In our case, the ability to simulate several regimes from bubbly to slug or churn flows induces some precise knowledge of the interfacial quantities. Dispersed field approaches require complex correlations to model interfacial area, especially for coalescence modelling. Therefore, having to resort to an interface locating method seems a wise choice. As our goal is to deal with several bubble scales, locating every interfaces with a good precision would require large mesh sizes and a great amount of CPU time. We developed an hybrid method, aiming at dealing with small and spherical bubbles with dispersed field modelling and simulating the interface of large and distorted ones (Eötvos number $>4$ ). Here, the idea is to couple the dispersed and the located approach to take advantage of their respective capabilities: the accuracy and the low CPU consuming of the dispersed approach, and the simulation of the distortion of the larger bubbles with a located interface model that avoids the use of a priori correlations on the bubble shapes. This naturally leads to a spatial filter scale depending on the flow properties. The dispersed approach for small interfaces that can be considered as spherical has already been validated [3] and has given reasonable agreement with experimental data, using the Eulerian multifield CFD code NEPTUNE_CFD.

As a consequence, we concentrate here our work on the simulation of large and deformed bubbles. A first step towards the hybrid method is to build a set of equations to model the large interface field. Numerical model and its application to bubble rising are summarized here and can be found in [4] for more details. After proving the efficiency of the method on the well-known bubble rising cases, we will test the model on two-bubble coalescence experimental cases. The predictions of the model on local coalescence cases have a key role in the validity of the extension of the interface locating model towards studying slug flows.

\section{STATE OF THE ART FOR INTERFACE TREATMENT AND COALESCENCE}

The rise of bubbles in viscous liquids/fluids remains a fundamental problem in fluid physics. Despite several theoretical [5], experimental [6] and over these recent years numerical [7, 8] studies, this paradigm is still a topic of major interest.

Most of the current techniques applied to interface flows such as large bubble rising have been developed taking into account two major aspects: capturing/tracking the interface and stabilizing the flow solver to handle discontinuous fluid properties at the interface.

Several categories of interface locating exist. The first one is Lagrangian methods. They can consist in Lagrangian grid methods [9] where the background grid is following the interface. The main limitation is that this method cannot track surfaces that break apart or intersect. Another kind of Lagrangian methods is the meshless approach like the smoothed particles hydrodynamics [10] but here the limit usually comes from the number of particles. The second category is the front tracking method [11]. The motion equation for the flow field is solved on a fixed grid, and the interface position is tracked explicitly by markers distributed evenly on the interface. Difficulty generally comes from the repartition of the markers, which should ideally be kept equidistant, especially for coalescence of interfaces' applications. The third category of these methods consists in capturing the interface 
using various volume functions defined on the fixed grid used to solve the governing equations of the flow. The volume of fluid (VOF) [12] method and level-set [13] approaches are part of this group. VOF methods are conservative, but interface properties such as normal and curvature are difficult to calculate accurately. Level-set methods automatically deal with topological changes but are not conservative. Several techniques have been developed to ensure volume conservation and improve the locating method, such as a combination of level-set and VOF method [14], or the augmented Lagrangian method coupled to VOF techniques [15].

Olsson and Kreiss [16] introduced a level-set method with an artificial compression step performed after the advection of the level-set function to ensure that the thickness of the transition layer is preserved.

In our work, the Eulerian approach is used. This approach of the two-fluid model allows the direct use of the volume fraction as an interface function. Thus, the equivalent to the level-set function is self-transported by the resolution of the momentum balance equation. To keep a good location of the interface over time, the same artificial compression step as Olsson and Kreiss is used.

Concerning coalescence, only few experiments provide results on local bubble coalescence such as the experiment of Chen et al., described in [17] and of Brereton and Korotney [18]. Numerical works on this phenomenon usually follow interface treatment methods and can be found with marker approach [19] and VOF approach [17, 20].

\section{MATHEMATICAL FORMULATION AND NUMERICAL METHOD}

\subsection{Governing equations}

The two-fluid model is based on Eulerian multifield balance equations. The so-called six equations model (three per field: mass, momentum and energy) is computed in NEPTUNE_CFD code, assuming that there is only one pressure in the system. The solver SIMPLE [21] is based on a finite volume discretization, together with a collocated arrangement for all variables. An iterative coupling of equations is used to ensure mass and energy conservation. The data structure is totally face-based, which allows the use of arbitrary-shaped cells including nonconforming meshes. In the cases presented in this paper, 2D axisymmetric and 3D square meshes are used. The notation $\Delta x$ in the paper represents the characteristic space dimension of these 2D and 3D square meshes. The discretization scheme is centred with a slope test to avoid over or under-shots on the volume fractions.

A full description of the model balance equations can be found in Ishii and Hibiki [22]. In the simplified case of isothermal two-fluid model, the system of equations can be written as mass and momentum balances:

$$
\begin{gathered}
\partial_{t}\left(\alpha_{k} \rho_{k}\right)+\partial_{x_{i}}\left(\alpha_{k} \rho_{k} U_{k, i}\right)=\Gamma_{k} \text { with } \sum_{k} \Gamma_{k}=0 \\
\partial_{t}\left(a_{k} \rho_{k} U_{k, i}\right)+\partial_{x_{i}}\left(\alpha_{k} \rho_{k} U_{k, i} U_{k, j}\right)=\partial_{x j}\left(\alpha_{k} \tau_{k, i j}\right)-a_{k} \partial_{x_{i}}(P)+\alpha_{k} \rho_{k} g_{i}+I_{k, i}
\end{gathered}
$$

where $\rho_{k}, U_{k}, a k, \Gamma_{k}$ and $\tau_{k, i j}$, and $I_{k, i}$ denote, respectively, the density, the mean velocity, the volumetric fraction, the mass transfer and the total stress tensor including laminar and 
turbulent contribution, the mean pressure and the interfacial momentum exchange between phases. In our cases, the Reynolds number is lower than 100, so we suppose the cell sizes small enough to simulate every space scale of the flow. Therefore, no turbulence model is used. The interfacial momentum exchange is constituted here by two interfacial forces: surface tension force and drag force. This later appears in momentum balance equations due to averaging. Its local sum over phases is equal to 0 and depends on the relative velocity between the phases

$$
\vec{F}_{\text {drag, } 1}=\frac{\alpha_{1} \alpha_{2}\left(\overrightarrow{v_{2}}-\overrightarrow{v_{1}}\right)\left(\alpha_{1} \rho_{1}+\alpha_{2} \rho_{2}\right)}{\tau}
$$

As we consider in this paper a local separated flow in the two-fluid model, the role of the drag force is to enforce a no slip condition and so equality of the phase velocities at the bubble interface. The closure law, eqn (3), is applied with a very short relaxation time $t$ to achieve this internal wall condition at the interface $(\tau=\Delta t / 100)$.

\subsection{Surface tension}

Large bubbles are no longer considered as a dispersed, but as a continuous field; therefore, the surface tension, eqn (4), has to be taken into account in the model. From an Eulerian point of view, a surface force has to be implemented in volume:

$$
\vec{F}_{\text {sta }}=\sigma K \vec{n}
$$

where $\sigma, k$ and $\vec{F}$ denote, respectively, the surface tension, the curvature and the normal vector. The curvature $k$ is defined as the divergence of the unit normal vector at the interface. A colour function $c$ locating the interface, such as a level-set function, is a descriptor of this normal vector. The unit normal vector and the curvature can be calculated with the colour function, obtained by diffusion of the volume fraction. Here it becomes important that the thickness of the interface is kept constant so that the colour function gives a good approximation of the local curvature. The continuum surface force method described by Brackbill et al. [23] allows the reformulation of the surface tension into an equivalent volume force, eqn (5), that can be added to the momentum balance equation. In the two-fluid model, this force is split between the two phases occupying the cell, since two momentum equations are solved. The average model is here the volume one

$$
\vec{F}_{s t}^{k}=a_{k} \sigma\left[\nabla \cdot\left(\frac{\nabla c}{\|\nabla c\|}\right)\right] \nabla c \mathrm{~d} V
$$

\subsection{Interface sharpening}

As already discussed in the previous section, the curvature calculation requires to pay some attention to the interface thickness. An artificial compression method coupled to a level-set method [16] improves the volume conservation. This interface sharpening method consists in resolving the following equation on a nonphysical time step between two physical steps to ensure the interface thickness to be kept constant. In our case, the volume conservation is verified in the resolution of the mass balance equation. Therefore, the main role of this 
artificial step is not to provide a good volume conservation but to keep the accuracy of interface quantities calculation constant over time

$$
\partial_{\tau}\left(a_{k}\right)+\nabla\left(a_{k}\left(1-a_{k}\right) \vec{n}\right)=\varepsilon \Delta \alpha_{k}
$$

The parameter $\varepsilon$ controls the final interface width at convergence and is here chosen so that the interface thickness is two cells. The nonphysical time $\Delta \tau$ is chosen to ensure CFL and Fourier numbers under 0.5 and to minimize the number of iteration leading to a steady interface width: $\varepsilon=\Delta x / 2$ and $\Delta \tau=\Delta x / 32$.

\section{APPLICATION TO BUBBLE RISING: RAYMOND AND ROSANT'S EXPERIMENT}

\subsection{Experimental description}

The capturing interface process described in the previous section is validated against the bubble rising experiments of Raymond and Rosant [6]. Bubbles are released in a stationary blend of water and glycerol at time $t=0 \mathrm{~s}$ in a rectangular Plexiglas tank of $0.3 \times 0.2 \mathrm{~m}^{2}$ inside cross-section and $0.5 \mathrm{~m}$ height. The reference pressure is the atmospheric pressure $(1013 \mathrm{hPa})$. The liquid physical properties (viscosity and density) are given in Table 1 .

Table 1: Fluid properties for the Raymond and Rosant's experiment [6].

\begin{tabular}{lccc}
\hline Series & Viscosity $(\mathrm{Pa} \mathrm{s})$ & Density $\left(\mathrm{kg} \mathrm{m}^{-3}\right)$ & Surface tension $\left(\mathrm{N} \mathrm{m}^{-1}\right)$ \\
\hline S1 & 0.687 & 1250 & 0.063 \\
S3 & 0.242 & 1230 & 0.063 \\
S5 + & 0.0733 & 1205 & 0.064 \\
S6 $\triangle$ & 0.0422 & 1190 & 0.064 \\
\hline
\end{tabular}

The final bubble velocity and shape (ratio of height $h$ to width $w$ of the bubble) are studied as functions of the bubble initial diameter $d_{\text {bubble }}$, ranging from 1 to $12 \mathrm{~mm}$. An axisymmetric geometry is used, reproducing the experiment situation of bubbles rising in pipe with a large section compared with the bubble diameters. The air density and viscosity are, respectively, $1.29 \mathrm{~kg} \mathrm{~m}^{-3}$ and $1.8410^{-5} \mathrm{~Pa}$ s. A Dirichlet's condition on the pressure field is imposed at the top boundary.

\subsection{Sensitivity analysis}

Sensitivity analysis, both on the size of the computational domain and the mesh refinement, has been carried out. Experimental data show that the wall effects on the bubbles can be considered as negligible. To optimize the CPU cost of the simulation, a sensitivity analysis on the computational domain is done to find the smallest possible computational domain in which walls have negligible effect on the terminal velocity and shape of the simulated bubbles.

Radius from two to six times the diameter of the initial bubble was tested for the simulation of the experimental case S5 (see Table 1) with a 8-mm diameter bubble. Figure 1 displays the terminal bubble shape for the tested computational domain size. The terminal velocity can be considered as independent from the domain size $D$ when $D>4 d_{\text {bubble }}$. A grid-independent 

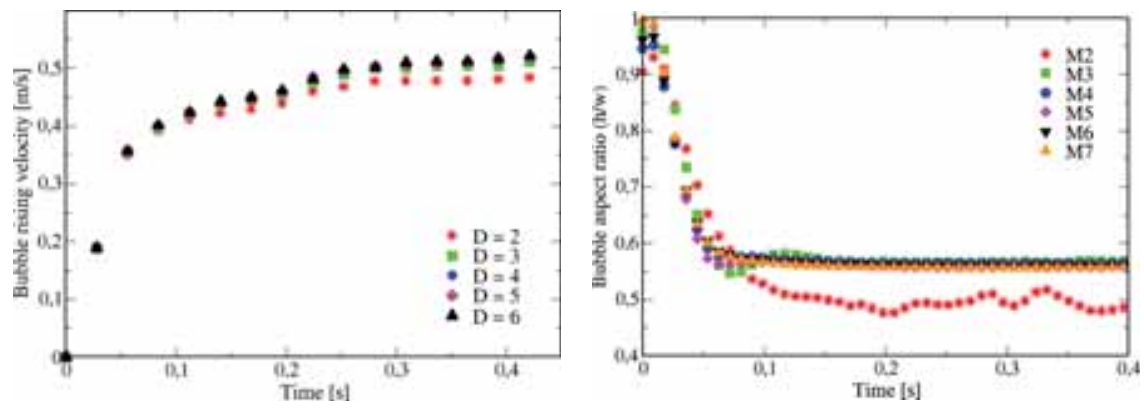

Figure 1: Sensitivity analysis on the computational domain size (on the left) and on the mesh refinement (on the right). $D$ is the computational domain radius, here expressed in bubble diameters. The different meshes are axisymmetric and square in the plane of the axis: M2 $\left(\delta=d_{\text {bubble }} / \Delta x=15\right)$, M3 $(\delta=20)$, M4 $(\delta=25)$, M5 $(\delta=30)$, M6 $(\delta=35)$ and M7 $(\delta=40)$.
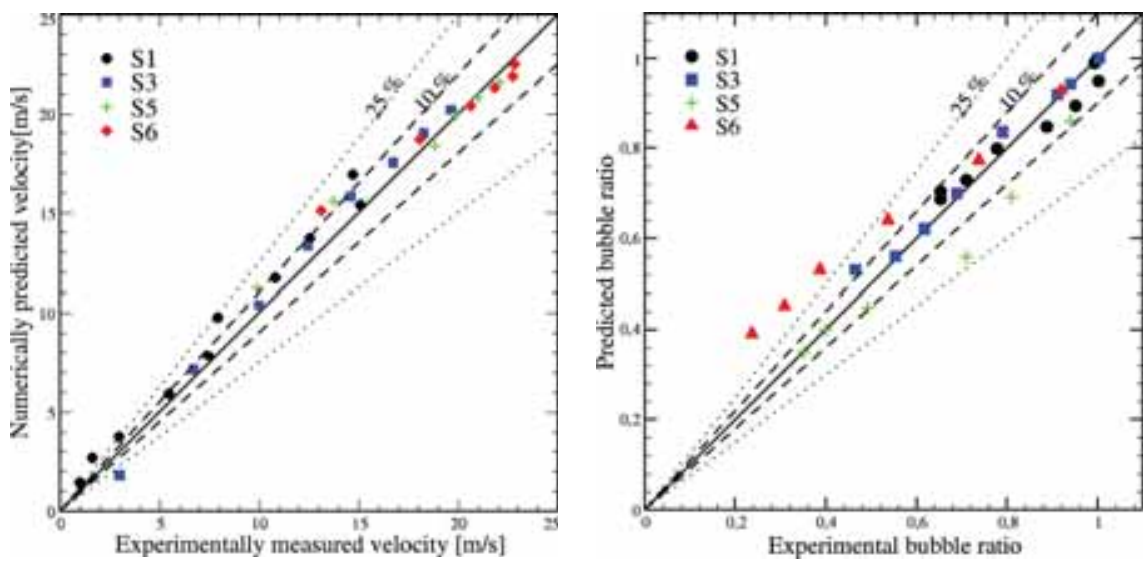

Figure 2: Comparison between the numerical predictions and the experimental measurements for the bubble terminal velocity (on the left) and aspect ratio (on the right) for various liquid properties (see Table 1). The dashed and dotted lines represent, respectively, $10 \%$ and $25 \%$ of discrepancy between calculations and measurements.

test was carried out on the axisymmetric rise of a single bubble in a liquid on six different meshes. The six grids are M2 $\left(\delta=\mathrm{d}_{\text {bubble }} / \Delta x=15\right)$, M3 $(\delta=20)$, M4 $(\delta=25)$, M5 $(\delta=30)$, M6 $(\delta=35)$ and M7 $(\delta=40)$. According to the previous sensitivity analysis on the domain size, the domain is chosen to have a 4-bubble diameter radius and 12-bubble diameter height. Figure 1 shows that the mesh M5 provides a nearly grid-independent solution. This grid will be used in the following.

\subsection{Model validation with experiments}

Figure 2 presents the comparison between numerical predictions and experimental measurements for the terminal velocities and shapes for the four experiments S1, S3, S5 and S6. 
The predicted velocities are found to be in a good agreement with the experimental data, the deviation being lower than $10 \%$. Concerning the bubble aspect ratio, as shown in Fig. 2, the deviation is lower than $10 \%$ for experiments S1 and S3, and lower than $23 \%$ for experiment S5. Only the three fastest bubbles are found to be not accurately predicted for the experiment $\mathrm{S} 6$, which is the less viscous case.

For these most distorted cases (experiment case $\mathrm{S} 6$ and bubble diameters $>6 \mathrm{~mm}$ ), a part of the gap between measurements and numerical predictions could be explained by a discrepancy between the initial conditions in the experiment and the simulation. These bubbles being the fastest, we can doubt that their initial shape is a perfect sphere in the experiment. As it has been shown in [7], an initial deformation of the bubble can impact the terminal shape and velocity of the bubble for small viscosities that may explain here part of the difference observed in the experiment S6 (red markers in Fig. 2).

\section{BUBBLE COALESCENCE}

The numerical predictions of the model for the terminal velocities and shapes prove to be in reasonable agreement with the experimental observations for bubble rising cases. Aiming at simulating slug flow regimes, the accuracy of local coalescence phenomenon prediction is of high concern. Two different experiments of local bubble coalescence are modelled here. The first one is described by Chen et al. [17]. The experiments of Brereton and Korotney [18] provide a good qualitative description of the coalescence of two bubbles rising in a quiescent liquid.

\subsection{Experiment of Chen et al.}

The experiment of Chen et al. [17] consists in the coalescence of two identical bubbles, shown in Fig. 3. The injection is a cylindrical nozzle of diameter $6 \mathrm{~mm}$. The liquid physical properties are $\rho_{1}=1220 \mathrm{~kg} \mathrm{~m}{ }^{-3}, \mu_{1}=0.11 \mathrm{~kg} \cdot \mathrm{m}^{-1} \cdot \mathrm{s}^{-1}$ with a surface tension coefficient $\sigma=0.066 \mathrm{~N} \cdot \mathrm{m}^{-1}$ between the water-glycerin blend and the air. These properties give the following Eötvos and Morton numbers: Eö $=5$, Mo $=4.1 \times 10^{-3}$ and a density ratio $\rho_{l} / \rho_{g} \approx 1000$. The experimental data consist in four pictures with a delay of $15 \mathrm{~ms}$ between each captures.

A first attempt is a numerical calculation with spherical shapes for bubble initialization. To respect the geometry of the experiment, the computational domain is $2 \mathrm{D}$ axisymmetric with a square grid. The results of the calculation are presented in Fig. 4. The time scale of the coalescence phenomenon seems shorter than in the experiment $(30 \pm 15 \mathrm{~ms}$ in experiment $v s$. $20 \pm 10 \mathrm{~ms}$ in the simulation). Grid dependency on the numerical results has been tested on three meshes; the differences between the coarse $\left(\delta=d_{\text {bubble }} / \Delta x=10\right)$, the medium $(\delta=20)$ and the fine $(\delta=30)$ grids are presented in Fig. 5. It shows that the medium and the fine grids predict nearly the same bubble shape. Therefore, the observed discrepancy between the numerical and the experimental results is not due to a lack of grid refinement. The reason may be the differences between initial conditions (gas inlet in the experiment compared with stationary spherical bubbles in the simulation).

A second simulation is conducted this time with an initialization of the bubble shapes derived from the first experimental picture at time $t=45 \mathrm{~ms}$ (time $t=0 \mathrm{~s}$ for the simulation). The same capture timing as in the experiment is presented in Fig. 6 and they are superposed on the experimental pictures. 


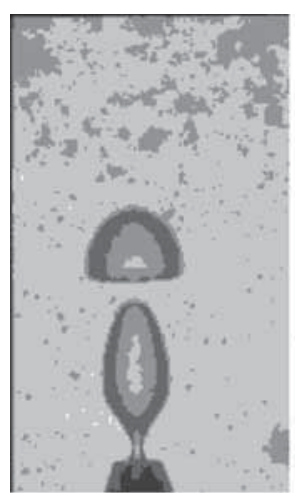

(a) $\mathrm{t}=45 \mathrm{~ms}$

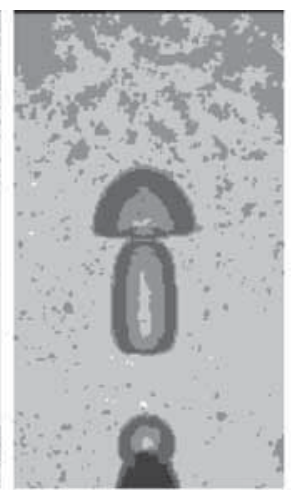

(b) $\mathrm{t}=60 \mathrm{~ms}$

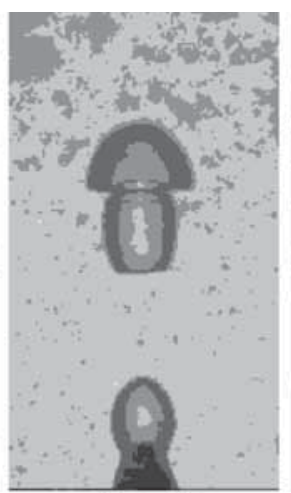

(c) $\tau=75 \mathrm{~ms}$

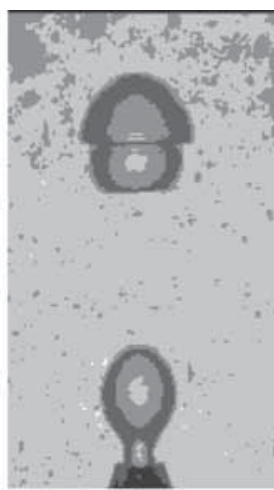

(d) $\tau=90 \mathrm{~ms}$

Figure 3: Experimental pictures of local coalescence experiment. The capture frequency is $15 \mathrm{~ms}$. The black nozzle at the bottom of each picture has a diameter of $6 \mathrm{~mm}$ (source [17]).

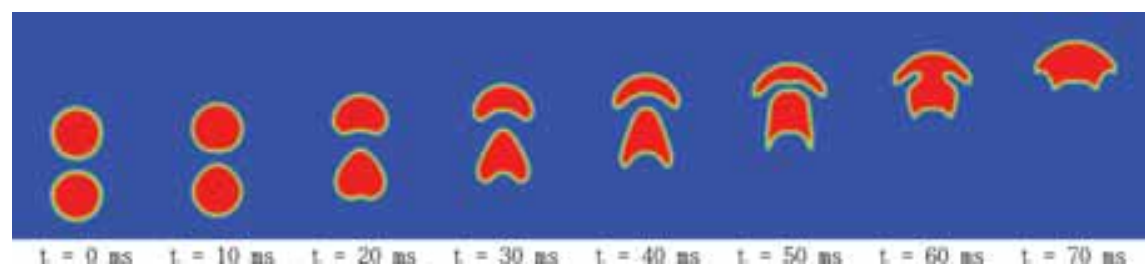

Figure 4: Numerical modelling of two-bubble in-line coalescence and the gas volume fraction are presented. The delay between two pictures is $10 \mathrm{~ms}$.

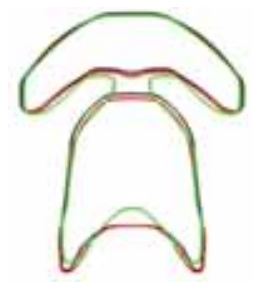

Figure 5: Void fraction contour $\alpha_{\text {bubble }}=0.5$ for three different meshes (coarse: green, medium: red, fine: black).

We can observe that with such initialization, the time scale of the phenomenon is better than with the initial spherical shapes. There is still differences between the bubble shapes and velocities, but this was expected since the experimental bubbles in Fig. 6 at time $t=45 \mathrm{~ms}$ already have a velocity, whereas the initial velocities are set to zero in the simulation. The velocity difference can be observed in Fig. 6, as the yellow line materializing the bottom of the calculation domain is rising over time and its velocity being directly linked to the delay between experimental and numerical bubbles. 


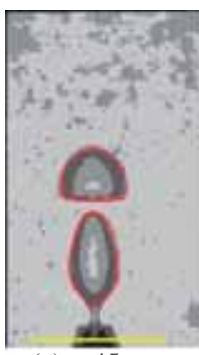

(a) $\mathrm{t}=45 \mathrm{~ms}$

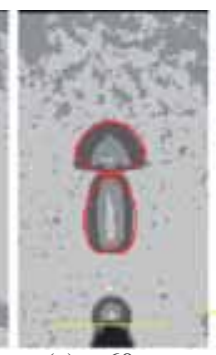

(a) $\mathrm{t}=60 \mathrm{~ms}$

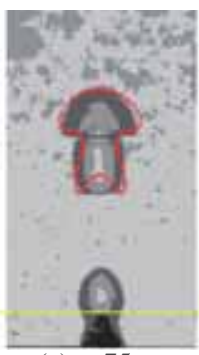

(c) $\tau=75 \mathrm{~ms}$

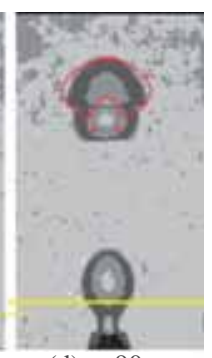

(d) $\tau=90 \mathrm{~ms}$

Figure 6: Superposition of the numerical simulation with the experimental pictures. The red line represents the void fraction contour $a_{\text {bubble }}=0.5$. The yellow line materializes the bottom of the calculation domain. The rise of this yellow line expressed the delay of the calculated bubbles compared with the experimental observations due to the initial condition of stationary bubbles at $t=0 \mathrm{~s}$.

\subsection{Brereton and Korotney's experiment}

Four cases of two-bubble coalescence are simulated: two cases of coaxial or in-line coalescence and two cases of oblique coalescence. Each type of coalescence is being treated in two dynamic conditions, described by Reynolds, Morton and Eötvos numbers: case (a) $(\operatorname{Re}=1$, $\mathrm{Mo}=30, \mathrm{Eö}=16)$ and case (b) $\left(\mathrm{Re}=43, \mathrm{Mo}=2 \times 10^{-4}\right.$, Eö = 16). Both the leading and the trailing bubbles have the same volume, with an initial bubble diameter of $1 \mathrm{~cm}$. The computational domain is a rectangular 3D box of size $0.04 \times 0.04 \times 0.08 \mathrm{~m}^{3}$ and the origin is placed at the centre of the bottom face. The initial bubbles have a diameter of $1 \mathrm{~cm}$ and are released from positions $\{(0,0,0.025)(0,0.08,0.125)\}$ and $\{(0,0,0.025)(0,0,0.125)\}$, respectively, for the oblique and the coaxial coalescence experiments. Regarding the discretization, the space scale is $\Delta x=5 \times 10^{-3} \mathrm{~m}$ for case (a) and $\Delta x=2.5 \times 10^{-3} \mathrm{~m}$ for case (b).

A comparison between numerical prediction and the experimental observations of the coalescence phenomena is presented in [4]. Indeed, the most viscous case (a) needs the finest grid because of the difficulty in reproducing the sharp tail of the second bubble due to viscous effects. The time scale of the coalescence is well predicted by the numerical simulations for all cases. According to the time difference between pictures, the duration of the coalescence phenomenon can be estimated at $75 \pm 15 \mathrm{~ms}$ for case (a) and $45 \pm 15 \mathrm{~ms}$ for case (b). Despite the complexity of the phenomenon, the shapes of both leading and trailing bubbles are in excellent agreement with the experimental observations [4].

A qualitative comparison of these bubble shapes is given in Fig. 7. The bubble ratio is again the ratio of the height to the width of the bubble. Since the bubble shapes are far from being always ellipsoidal, two measures are given for each bubble. The first one, represented in Fig. 7 by the hollow symbols, is obtained by a square assumption for the bubble ratio calculation, and the second one represented by the solid symbols, is obtained with an ellipsoidal assumption. Except for the case of oblique coalescence in the less viscous liquid (b) $(\operatorname{Re}=43$, $\mathrm{Mo}=2 \times 10^{-4}$ and Eo $=16$ ), represented by the red triangle markers in Fig. 7, we mainly have a prediction of the bubble shapes during the coalescence phenomenon with an error lower than $25 \%$. The differences are mostly due to the difference between experimental and 


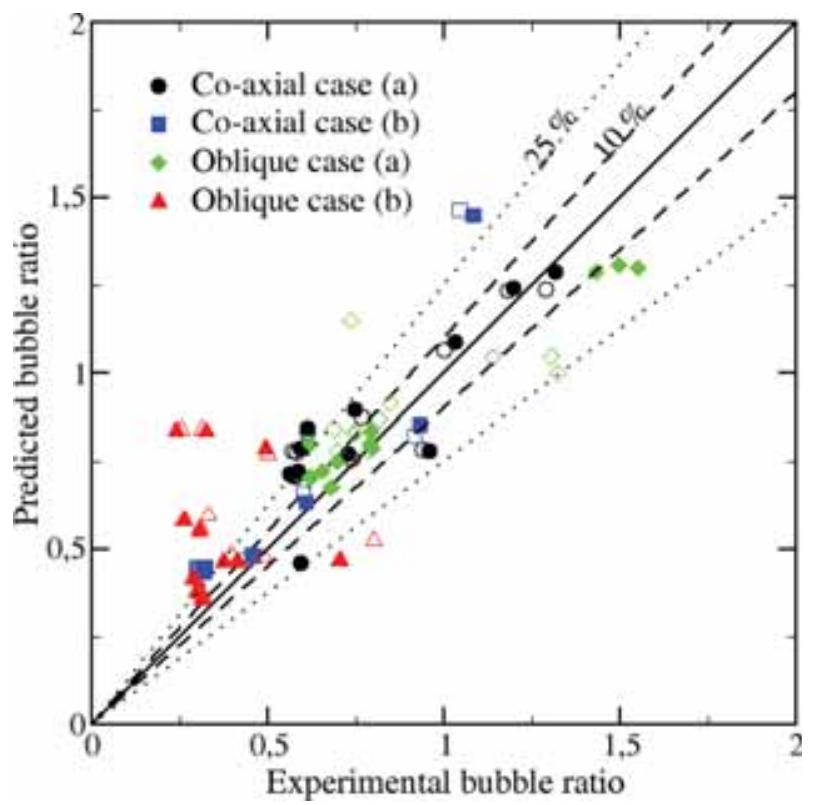

Figure 7: Comparison between the experimental bubble ratio from Brereton and Korotney [18] and numerical predictions of the four cases of coalescence previously described (coaxial and oblique). The hollow symbols represent bubble ratio evaluation with a squared approximation and the solid symbols an ellipsoidal approximation of the bubble shape. The dashed and dotted lines represent, respectively, $10 \%$ and $25 \%$ of discrepancy between prediction and measurements.

numerical initial conditions. Indeed, the simulations are initiated with two spherical stationary bubbles, as the trailing bubble is injected in the domain while the leading bubble is already rising in the experiments.

\section{CONCLUSION}

The dynamics of a deformed bubble has been studied using a two-fluid model adapted to cases with located interfaces. Experimental validations have been carried out for singlebubble rising and two-bubble coalescence cases. Quantitatively for bubble rising, the predicted terminal shape and the velocity of the bubbles are in reasonable agreement with the experiments of Raymond and Rosant [6]. Qualitatively for two-bubble coalescence, the coalescence time scale and bubble behaviour are found to be quite similar to both Chen et al. [17] and Brereton and Korotney's experiments [18]. The model dedicated to large distorted bubbles was successfully applied to coalescence cases in different liquid conditions and gives good agreement between the experimental bubble shapes and the numerical predictions. This is comforting us in the way of a hybrid three-field model to address regime transitions such as bubbly to slug flow, as one of the main incertitude in today's dispersed models is the efficiency of coalescence correlations when the bubble diameter is increasing and the bubble deforming. 


\section{ACKNOWLEDGEMENTS}

This work has been achieved in the framework of the NEPTUNE project, financially supported by CEA (Commissariat a l'Energie Atomique et aux Energies Alternatives), EDF (Electrité de France), IRSN (Institut de Radioprotection et de Sûret'e Nuclaire) and AREVA-NP, in collaboration with I2M laboratory (Bordeaux, France).

\section{REFERENCES}

[1] Weisman, J. \& Kang, S.Y., Flow pattern transitions in vertical and upwardly inclined lines. International Journal of Multiphase Flow, 7, pp. 271-291, 1981. doi: http:// dx.doi.org/10.1016/0301-9322(81)90022-7

[2] Bestion, D., Applicability of two-phase CFD to nuclear reactor thermalhydraulics and elaboration of best practice guidelines. Nuclear Engineering and Design, 253, pp. 311-321, 2012. doi: http://dx.doi.org/10.1016/j.nucengdes.2011.08.068

[3] Mimouni, S., Archambeau, F., Boucker, M., Laviéville, J. \& Morel, C., A second order turbulence model based on a Reynolds stress approach for two-phase boiling flow and application to fuel assembly analysis. Nuclear Engineering and Design, 240, pp. 2225-2232, 2009. doi: http://dx.doi.org/10.1016/j.nucengdes.2009.11.020

[4] Denefle, R., Mimouni, S., Caltagirone, J.P. \& Vincent, S., Multifield hybrid approach for bubble to slug flow transition modelling. Proceedings of AFM2012: 9th International Conference on Advances in Fluid Mechanics, Split, Croatia, 2012.

[5] Clift, R., Grace, J.R. \& Weber, M.E., Bubbles, Drops, and Particles, Academic Press: New York, 1978.

[6] Raymond, F. \& Rosant, J.M., A numerical and experimental study of the terminal velocity and shape of bubbles in viscous liquids. Chemical Engineering Science, 55, pp. 943-955, 2000. doi: http://dx.doi.org/10.1016/S0009-2509(99)00385-1

[7] Hua, J. \& Lou, J., Numerical simulation of bubble rising in viscous liquid. Journal of Computational Physics, 222, pp. 769-795, 2007. doi: http://dx.doi.org/10.1016/j. jcp.2006.08.008

[8] Koebe, M., Bothe, D. \& Warnecke, H.J., Direct simulation of air bubbles in water/glycerol mixtures: shapes and velocity fields. Proceedings of FEDSM'03: 4th ASMEJSME Joint Fluids Engineering Conference, Honolulu, Hawaii, USA, 2003.

[9] Hyman, J.M., Numerical methods for tracking interfaces. Physica D, 12, pp. 396-407, 1984. doi: http://dx.doi.org/10.1016/0167-2789(84)90544-X

[10] Gingold, R.A. \& Monaghan, J.J., Smoothed particle hydrodynamics - theory and application to non-spherical stars. Monthly Notices of the Royal Astronomical Society, 181, pp. 375-389, 1977.

[11] Unverdi, S.O. \& Tryggvason, G., A front-tracking method for viscous, incompressible, multi-fluid flows. Journal of Computational Physics, 100, pp. 25-37, 1992. doi: http:// dx.doi.org/10.1016/0021-9991(92)90307-K

[12] Hirt, C.W. \& Nichols, B.D., Volume of fluid (VOF) method for the dynamics of free boundaries. Journal of Computational Physics, 39, pp. 201-225, 1981. doi: http:// dx.doi.org/10.1016/0021-9991(81)90145-5

[13] Osher, S. \& Sethian, J.A., Fronts propagating with curvature-dependent speed: algorithms based on Hamilton-Jacobi formulations. Journal of Computational Physics, 79, pp. 12-49, 1988. doi: http://dx.doi.org/10.1016/0021-9991(88)90002-2

[14] Sussman, M. \& Puckett, E., A coupled level set and volume-of-fluid method for computing 3D and axisymmetric incompressible two-phase flows. Journal of Computational Physics, 162, pp. 301-337, 2000. doi: http://dx.doi.org/10.1006/jcph.2000.6537 
[15] Vincent, S., Caltagirone, J.P., Lubin, P. \& Randrianarivelo, T.N., An adaptative augmented Lagrangian method for three-dimensional multimaterial flows. Computers and Fluids, 33, pp. 1273-1289, 2004. doi: http://dx.doi.org/10.1016/j.compfluid.2004.01.002

[16] Olsson, E. \& Kreiss, G., A conservative level set method for two phase flow. Journal of Computational Physics, 210, pp. 225-246, 2005. doi: http://dx.doi.org/10.1016/j. jcp.2005.04.007

[17] Chen, L., Li, Y. \& Manasseh, R., The coalescence of bubbles - a numerical study. Proceedings of Third International Conference on Multiphase Flow: ICMF'98, Lyon, France, 1998.

[18] Brereton, G. \& Korotney, D., Coaxial and oblique coalescence of two rising bubbles. Proceedings of AMD-Vol. 119: The ASME Applied Mechanics Conference, Colombus, OH, USA, 1991.

[19] Singh, R. \& Shyy, W., Three-dimensional adaptive grid computation with conservative, marker-based tracking for interfacial fluid dynamics. 44th Aerospace Science Meeting, Reno, MV, USA, 2006.

[20] van Sint Annaland, M., Deen, N. \& Kuipers, J., Numerical simulation of gas bubbles behaviour using a three-dimensional volume of fluid method. Chemical Engineering Science, 60, pp. 2999-3011, 2005. doi: http://dx.doi.org/10.1016/j.ces.2005.01.031

[21] Patankar, S.V. \& Spalding, D.B., A calculation procedure for heat, mass and momentum transfer in three-dimensional parabolic flows. International Journal of Heat and Mass Transfer, 15, pp. 1787-1806, 1972. doi: http://dx.doi.org/10.1016/00179310(72)90054-3

[22] Ishii, M. \& Hibiki, T., Thermo-fluid Dynamics of Two Phase Flow, Springer-Verlag, 2006. doi: http://dx.doi.org/10.1007/978-0-387-29187-1

[23] Brackbill, J., Kothe, D. \& Zemach, C., A continuum method for modeling surface tension. Journal of Computational Physics, 100, pp. 335-354, 1992. doi: http://dx.doi. org/10.1016/0021-9991(92)90240-Y 